\title{
Características Físicas e Anatômicas de Cedrela odorata L. e Cedrelinga cateniformis Ducke
}

\author{
Neila Cristina Lima Fernandes ${ }^{1}$, Mara Lúcia Agostini Valle ${ }^{2}$, \\ Claudene Menezes Atayde Calderon ${ }^{1}$
}

${ }^{1}$ Centro Multidisciplinar - CMULTI, Universidade Federal do Acre - UFAC, Campus Floresta, Cruzeiro do Sul/AC, Brasil ${ }^{2}$ Centro de Formação em Ciências e Tecnologias Agroflorestais, Universidade Federal do Sul da Bahia - UFSB, Campus Jorge Amado, Itabuna/BA, Brasil

\begin{abstract}
RESUMO
Estudos de anatomia e pesquisas tecnológicas são necessários para as espécies Cedrela odorata e Cedrelinga cateniformis, uma vez que são muito utilizadas no setor moveleiro do município de Cruzeiro do Sul - Acre. Para avaliar o emprego adequado da madeira dessas espécies na indústria moveleira, este trabalho objetivou determinar suas propriedades físicas e anatômicas. Foram realizados ensaios para determinação da densidade básica e variação dimensional, bem como descrição macroscópica da madeira. Quanto aos fatores de anisotropia de contração e inchamento as espécies, foram classificadas como excelentes, com características adequadas para produção de móveis como estantes, mesas e armários.
\end{abstract}

Palavras-chave: propriedades anatômicas, propriedades físicas, densidade e estabilidade dimensional.

\section{Physical and Anatomical Characteristics of Cedrela odorata L. and Cedrelinga cateniformis Ducke}

\begin{abstract}
Studies of anatomy and technological research are needed for Cedrela odorata and Cedrelinga cateniformis species, since they are widely used in the furniture sector of Cruzeiro do Sul - Acre, Brazil. Thus, to enable the appropriate use of these wood species in the furniture industry, this study aimed to evaluate their physical and anatomical characteristics. Assays were performed to determine the basic density, dimensional variation and macroscopic description timber. The species were classified as lightweight and with excellent anisotropic and swelling factors, characteristics indicated for production of furniture as shelves, tables and cabinets.
\end{abstract}

Keywords: physical properties, anatomical properties, density and dimensional stability.

\section{INTRODUÇÃO}

A Região Amazônica é rica em espécies florestais, contudo, o maior obstáculo para a melhor utilização e aproveitamento da madeira de espécies amazônicas ainda éo desconhecimento de suas características tecnológicas, fisiológicas e morfológicas (Ferreira et al., 2004).
A falta de conhecimento das propriedades tecnológicas de grande parte das espécies arbóreas nativas da Amazônia acarreta uma exploração descontrolada de algumas (Carvalho et al., 2009) e um desinteresse comercial por outras. De acordo com Razera (2005), 
a pouca divulgação das características tecnológicas da madeira contribui para sua desvalorização e acarreta baixo índice de uso e inadequada aplicação da madeira.

A árvore, por ser proveniente de um sistema biológico complexo, apresenta propriedades anatômicas, físico-químicas e mecânicas que variam de espécie para espécie e de uma parte à outra na mesma árvore (Marcarti, 1992).

O estudo de sua estrutura anatômica faz-se importante, pois cada espécie possui estruturação anatômica única e diferente (Alves et al., 2012), na qual sua disposição, arranjo, proporção e dimensões relativas influenciam diretamente na densidade, resistência mecânica, trabalhabilidade, durabilidade natural e permeabilidade da madeira (Mafra, 1994).

Além das propriedades anatômicas, é importante avaliar as inter-relações existentes entre densidade, umidade, retratibilidade e expansão da madeira (Rezende et al., 1995), e os processos envolvidos durante sua transformação em produtos industrializados (Gatto et al., 2004), e assim direcionar à sua correta utilização e viabilizar um uso mais racional (Dias \& Lahr, 2004).

O baixo quantitativo de trabalhos de pesquisa sobre densidade e teor de umidade de madeiras exploradas comercialmente na Amazônia deixam lacunas significativas nas informações sobre madeiras amazônicas (Nogueira et al., 2008), bem como nas características anatômicas e comportamento dessas madeiras nos processos industriais.

$\mathrm{Na}$ Amazônia, pesquisas sobre características tecnológicas da madeira mostram que, mesmo existindo madeiras com propriedades semelhantes ao cedro, angelins, mogno, sucupiras, andiroba, louro, dentre outras, as serrarias persistem em comercializar essas mesmas espécies (Catanhede et al., 1997).

No Acre, o potencial de uso no setor madeireiro é de 607 espécies florestais (Araújo \& Silva, 2000), mas notabilizam-se, desde a década de 1980, basicamente 10 espécies como responsáveis pelo que corresponde a $84 \%$ do volume processado pelas indústrias de madeira (Acre, 1999).

Araújo (2002) aponta que, após extenso levantamento na literatura nacional e internacional acerca das propriedades físicas e mecânicas da madeira de 197 espécies ocorrentes em uma área florestal no estado do Acre, encontraram-se $37,5 \%$ com nome científico completo no nível gênero e espécie, 57,4\% somente no nível de gênero, e 5,1\% não constavam na literatura, não identificados, tampouco lhes indicando finalidade por falta de classificação, o que leva a concluir que ainda é pouco o conhecimento acerca das espécies florestais com potencial madeireiro no estado.

No município de Cruzeiro do Sul, estado do Acre, são escassos os estudos sobre as espécies madeireiras usadas na fabricação de móveis, inviabilizando assim, nesta região, o uso de algumas espécies madeireiras por falta de informações tecnológicas sobre elas (Calderon, 2012). Com isso, o estudo de características gerais, como massa específica aparente, aspectos estéticos e propriedades organolépticas, é de fundamental importância para melhor identificação das madeiras (Alves et al., 2012).

Panshin \& Zeeuw (1980) e Dias \& Lahr (2004) destacam que madeiras recomendadas à fabricação de móveis devem apresentar boa estabilidade dimensional e boa trabalhabilidade, para que possam durante a etapa de pintura, colagem e envernizamento, apresentar um acabamento superficial de qualidade. $O$ setor moveleiro do município de Cruzeiro do Sul trabalha com 30 espécies de madeira (Calderon, 2012). Deste quantitativo, as espécies Cedrelinga cateniformis Ducke e Cedrela odorata L., popularmente nominadas de cedro-água e cedro-rosa, respectivamente, por se destacarem pela boa trabalhabilidade, são de grande interesse para a indústria moveleira local, sendo, portanto, necessário descrever suas características organolépticas, anatômicas e propriedades físicas.

Em razão do baixo quantitativo de informações a respeito das espécies de madeira comercializadas atualmente pelo setor moveleiro do município de Cruzeiro do Sul, e com vistas a colaborar com a correta identificação dessas madeiras pelo próprio comércio de produtos florestais do município, este estudo teve como objetivo descrever as madeiras de Cedrela odorata L. e Cedrelinga cateniformis Ducke quanto à anatomia macroscópica e propriedades físicas.

\section{MATERIAL E MÉTODOS}

O estudo foi desenvolvido no município de Cruzeiro do Sul, localizado a oeste do estado do Acre, distante cerca de 710 quilômetros da cidade de Rio Branco, capital do Estado.

O município é caracterizado pela predominância de grandes rios e vasta extensão de floresta nativa. 
Conforme a classificação de Köppen, o clima do local é classificado como tropical úmido Af, com chuvas bem distribuídas durante o ano e com ausência de estação seca. Os índices pluviométricos variam de 1.600 a $2.750 \mathrm{~mm}$ ao ano. As temperaturas médias anuais são elevadas, variando entre $24,5{ }^{\circ} \mathrm{C}$ e $32^{\circ} \mathrm{C}$, com $85 \%$ de umidade relativa do ar.

As coletas do material foram realizadas em marcenarias existentes no Polo Moveleiro de Cruzeiro do Sul - Acre, sendo posteriormente levadas ao laboratório de Macroscopia da Universidade Federal do Acre, no Campus Floresta, para os demais procedimentos.

As espécies Cedrelinga cateniformis Ducke e Cedrela odorata L. foram escolhidas para o estudo, por serem de grande interesse econômico e muito utilizadas pelo setor moveleiro local. Uma semana após os pranchões de madeiras terem sido entregues nas marcenarias, foram obtidas amostras para determinação das propriedades físicas e anatômicas.

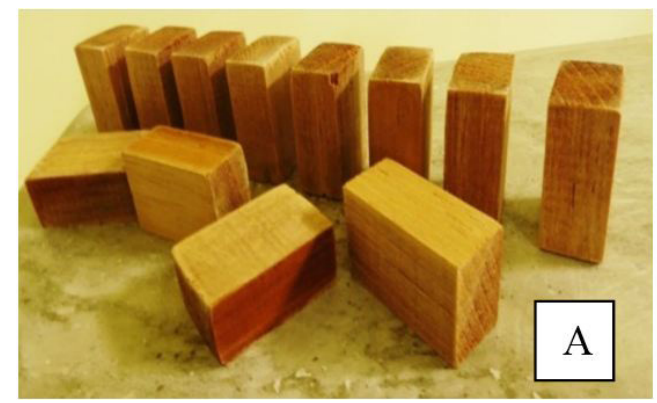

Os corpos de prova selecionados estavam isentos de defeitos, devidamente orientados e obtidos de regiões afastadas das extremidades das peças (Figura 1).

A identificação das espécies foi feita a partir da comparação com literatura específica, e em seguida amostras do material foram registradas na xiloteca desta Universidade no Laboratório de Anatomia, Tecnologia e Identificação da Madeira - LabMad.

A descrição anatômica macroscópica foi realizada de acordo com procedimento descrito por Coradin \& Muniz (1992), com auxílio de uma lupa de 10x de aumento, bisturi no 4 , lâminas $\mathrm{n}^{\circ} 22$ e 24 , pequena escova com cerdas médias e lixas de mão com granulação variada.

Os corpos de prova de 2,0 $\mathrm{cm} \mathrm{x} \mathrm{2,0} \mathrm{cm} \times 3,0 \mathrm{~cm}$ de largura, espessura e comprimento, respectivamente tiveram sua seção transversal lixada para melhor visibilidade dos elementos macroscópicos da madeira (Figura 2).

Posteriormente às análises macroscópicas, as amostras foram fotografadas com o auxílio de uma

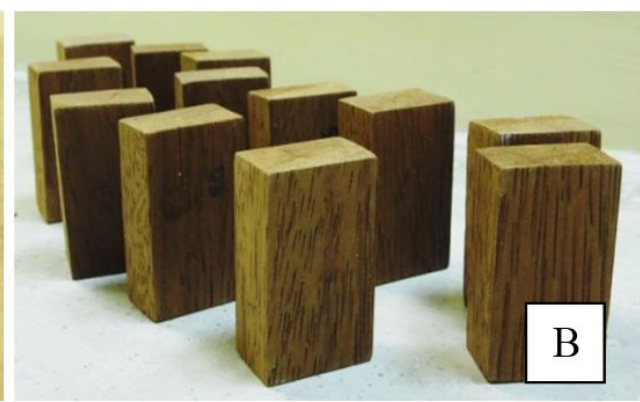

Figura 1. Corpos de prova das espécies Cedrela odorata (A) e Cedrelinga cateniformis (B). Figure 1. Specimens of Cedrela odorata (A) and cateniformis Cedrelinga (B) species.
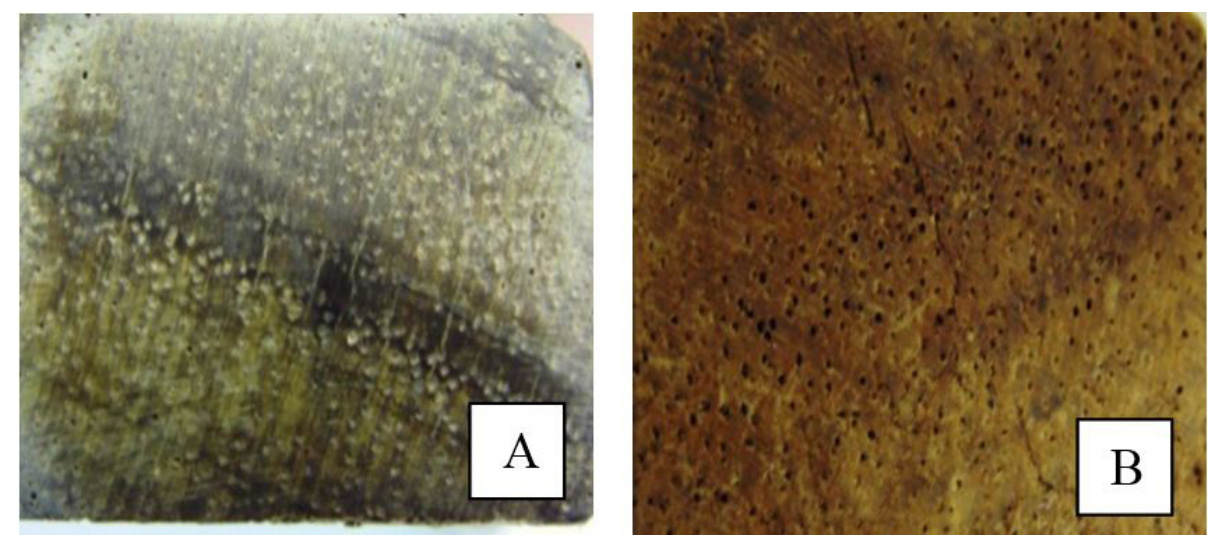

Figura 2. Plano transversal das espécies Cedrela odorata (A) e Cedrelinga cateniformis (B).

Figure 2. Transversal plane of the Cedrela odorata (A) and Cedrelinga cateniformis (B) species. 
câmera digital, modelo Moticam 2000-2.0MP Live Resolution, acoplada a um estereomicroscópio ótico, modelo Motic, com capacidade de aumento até 40x.

Os ensaios para determinação das propriedades físicas, tais como teor de umidade densidade básica e variação dimensional (contração e inchamento linear), foram realizados de acordo com a norma NBR 7190 (ABNT, 1997) (Figura 3).

A umidade foi determinada com base no método de pesagem em balança semianalítica, com precisão de 0,001g. Para determinação da densidade básica, utilizou-se o método estereométrico, e os corpos de prova ficaram imersos em água por aproximadamente 2 meses e meio até sua saturação. Para determinação da variação dimensional (contração e inchamento), mediu-se o aumento e diminuição do volume de cada amostra nas três direções estruturais, com auxílio de um paquímetro digital, à medida que o corpo de prova foi ganhando e perdendo umidade. A partir da variação dimensional de contração e inchamento existente entre

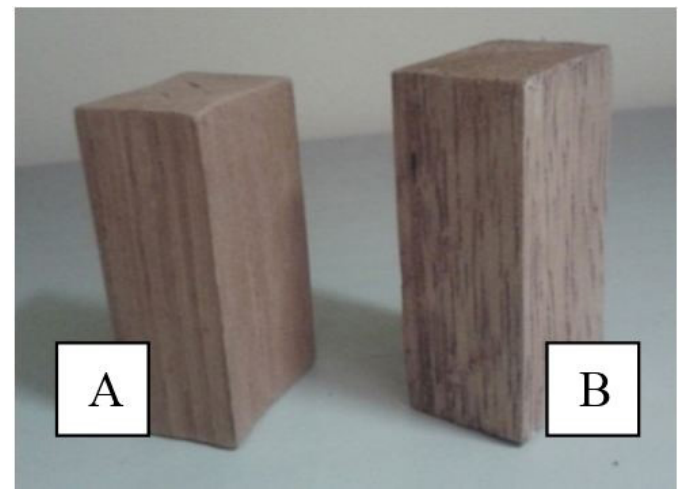

Figura 3. Corpo de prova das espécies para os ensaios físicos de Cedrela odorata (A) e Cedrelinga cateniformis (B). Figure 3. Specimen of the species for physical testing of Cedrela odorata (A) and Cedrelinga cateniformis (B). a direção tangencial e radial (T/R), foi determinado o Fator de Anisotropia de Contração (FA) e o Fator de Anisotropia de Inchamento (FI). Para auxiliar a análise deste resultado, foi utilizada a Tabela 1 descrita por Logsdon et al. (2008) em que os autores correlacionam o coeficiente de anisotropia com a qualidade da madeira para usos específicos.

\section{RESULTADOS E DISCUSSÃO}

\subsection{Caracterização anatômica}

A descrição anatômica macroscópica da madeira das duas espécies estudadas estão descritas abaixo:

\subsection{Cedrelinga cateniformis Ducke}

No plano transversal visível somente sob lente de 10x, apresentou: Parênquima axial: difuso, vasicêntrico, aliforme e confluente; Porosidade: difusa, presença de vasos solitários e múltiplos e presença de tilos (Figura 4); Cor: madeira apresenta coloração morrom-avemelhada a cinza-rosado; Camada de crescimento: pouco distintas. Nos planos tangencial e radial, observou-se frequência de série de células septadas sem conteúdo, um caráter típico para esta espécie. Os raios podem ser visto a olho nu não estratificado, e as linhas vasculares são retilíneas e obstruídas (Figura 5). A Figura 6 mostra os três planos de corte da espécie.

\subsection{Cedrela odorata L.}

No plano transversal, visível somente sob lente 10x, apresentou: Parênquima axial: paratraqueal vasicêntrico e marginal (Figura 7); Porosidade: poros solitários e geminados, obstruídos por uma substância branca difusa; Cor: madeira apresenta coloração marrom e vermelho; Camada de crescimento: distintos, marcado por parênquima terminal, porosidade em anel. No plano

Tabela 1. Coeficiente de anisotropia dimensional, qualidade e uso da madeira.

Table 1. Dimensional anisotropy coefficient, quality and use of wood.

\begin{tabular}{|c|c|c|c|}
\hline \multicolumn{2}{|c|}{ Coeficiente de Anisotropia } & \multirow{2}{*}{$\begin{array}{l}\text { Qualidade da } \\
\text { madeira }\end{array}$} & \multirow{2}{*}{ Indicação de uso para a madeira } \\
\hline Contração & Inchamento & & \\
\hline Até 1,50 & Até 1,54 & Excelente & $\begin{array}{l}\text { Móveis finos, esquadrias, barcos, aparelhos musicais, aparelhos } \\
\text { esportivos, etc. }\end{array}$ \\
\hline $1,50-2,00$ & $1,54-2,10$ & Normal & Estantes, mesas, armários, usos que permitam pequenos empenamentos. \\
\hline$>2,00$ & $>2,10$ & Ruim & $\begin{array}{l}\text { Construção civil (desde que observadas as características mecânicas), } \\
\text { carvão, lenha, etc. }\end{array}$ \\
\hline
\end{tabular}



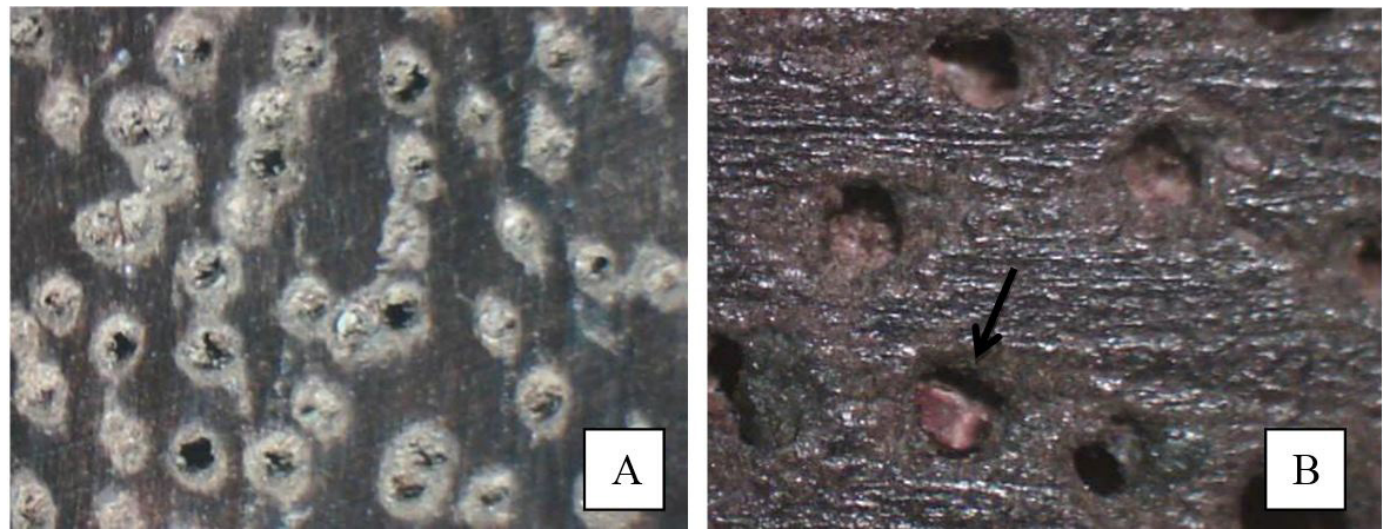

Figura 4. Corte transversal (aumento 40x) mostrando o parênquima (A) e presença de tilo (B) em Cedrelinga cateniformis. Figure 4. Traversal section (magnification 40x) showing the parenchyma (A) and the presence of methyl (B) of Cedrelinga cateniformis.
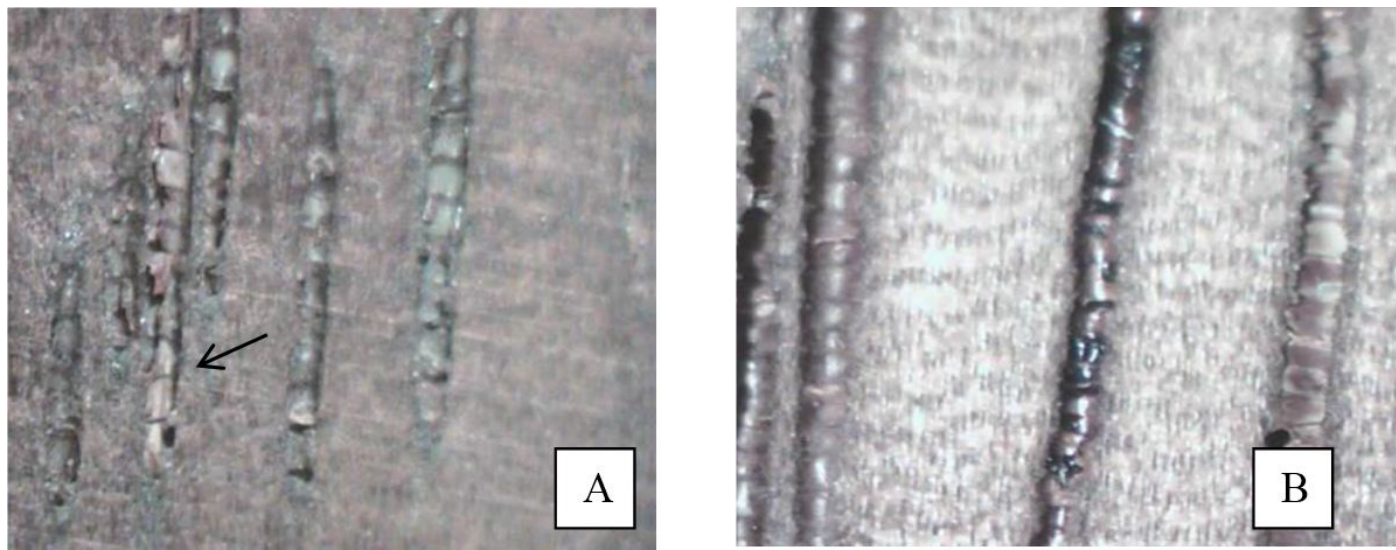

Figura 5. Plano longitudinal tangencial da madeira Cedrelinga cateniformis (aumento 40x). Fibras septadas (A) e raios e linhas vasculares (B).

Figure 5. Tangential longitudinal plane of wood Cedrelinga cateniformis. (magnification 40x). Septate fibers (A) and vascular ray and lines (B).
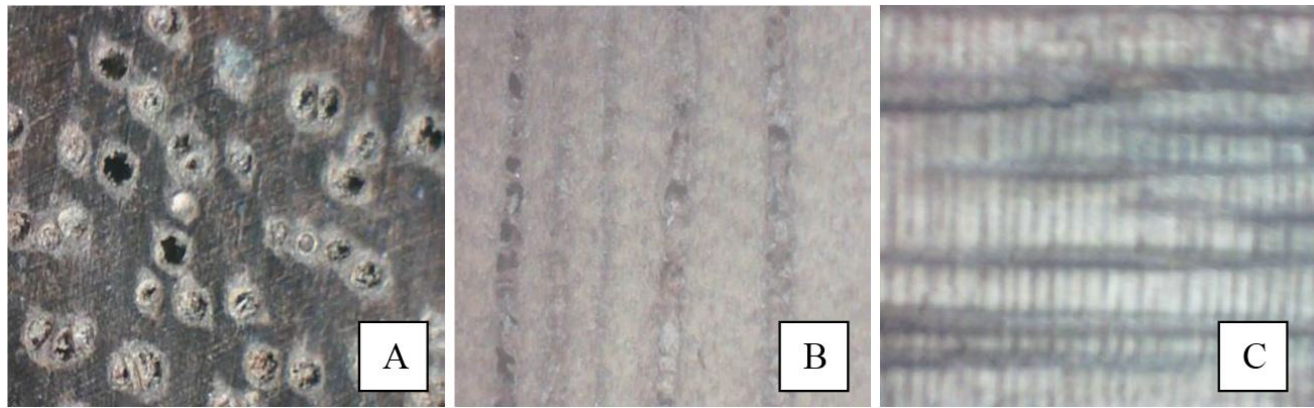

Figura 6. Estrutura macroscópica da madeira de Cedrelinga cateniformis (aumento 40x). Seção transversal (A); Seção longitudinal tangencial (B); Seção longitudinal radial (C).

Figure 6. Macroscopic structure of wood Cedrelinga cateniformis (magnification 40x). Cross section (A); Tangential longitudinal section (B); Radial longitudinal section (C). 

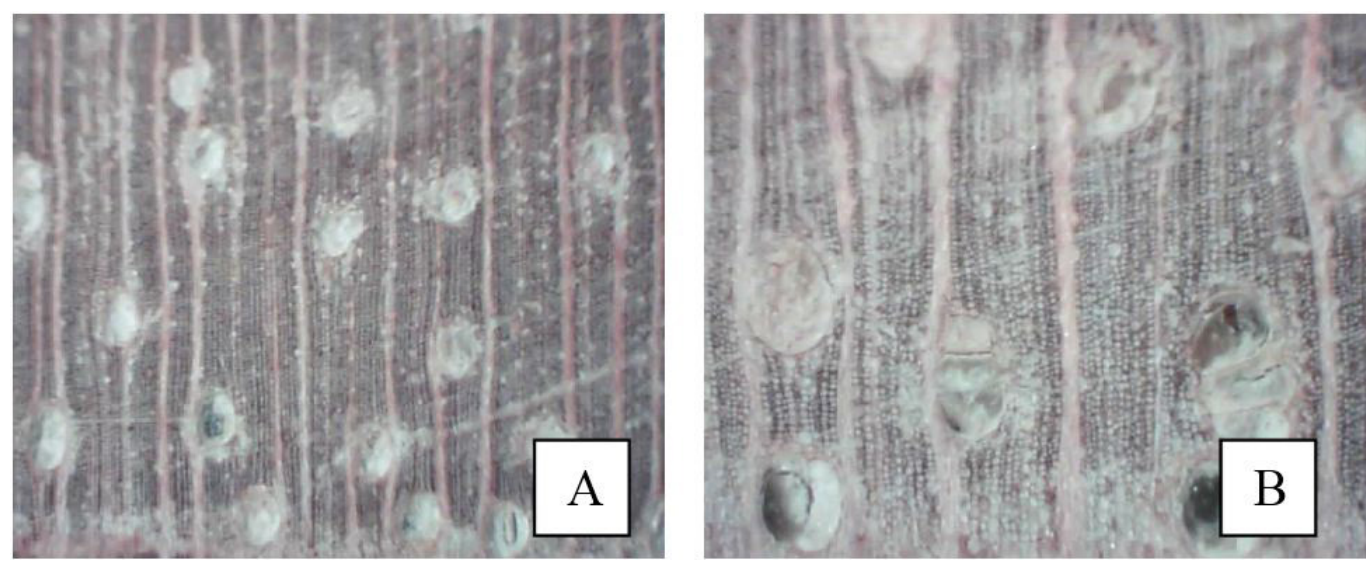

Figura 7. Plano transversal de Cedrela odorata evidenciando parênquima paratraqueal vasicêntrico marginal (A) e poros solitários e geminados (B) (aumento 40x).

Figure 7. Cedrela odorata cross plan showing marginal parenchyma paratracheal vasicentric (A) and solitary and twin pores (B) (magnification 40x).

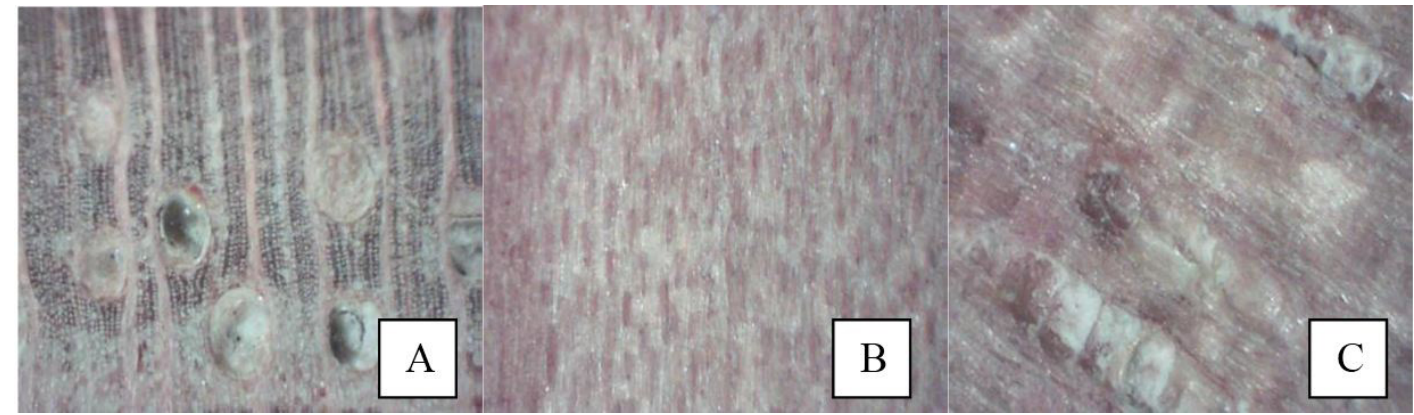

Figura 8. Estrutura macroscópica da madeira de Cedrela odorata. Seção transversal (A); Seção longitudinal tangencial (B); Seção longitudinal radial (C) (aumento 40x).

Figure 8. Macroscopic structure of wood Cedrela odorata. Cross section (A); Tangential longitudinal section (B); Radial longitudinal section (C) (magnification 40x).

radial, os raios só puderam ser observados com lente de aumento de 10x, e no plano tangencial apenas com aumento de 20x. As linhas vasculares são facilmente observadas e longas. Canais intercelulares longitudinais são frequentes, contendo, por vezes, resina (Figura 8).

\subsection{Caracterização física}

\subsubsection{Teor de umidade}

A umidade encontrada no lote para a espécie de Cedrela odorata foi 15,98\%, e para a espécie de Cedrelinga cateniformis foi de 16,58\%. "A determinação do teor de umidade e de sua variação no tronco das árvores ou das peças de madeira é de extrema importância no seu desempenho e utilização." (Oliveira et al., 2005, p. 116), como, por exemplo, em programas de secagem ou separar o material mais homogêneo quanto a este parâmetro, assim evitando defeitos nas peças de madeira durante sua secagem.

Quanto ao teor de umidade, as madeiras podem ser classificadas em comercialmente secas, quando o seu teor de umidade está entre $18 \%$ e $22 \%$; denominam-se secas ao ar, quando a sua umidade está entre 13\% e 17\%; e secas em estufa, quando atingem valores inferiores aos citados (Carvalho, 1996). Porém, o teor de umidade depende muito do local onde as madeiras estão sendo secas, pois quanto maior a umidade relativa do ar, mais alto também será este valor.

A umidade, apesar de não ser considerada uma característica intrínseca da madeira, trata-se de um parâmetro que afeta o comportamento do material quanto à trabalhabilidade, estabilidade dimensional, 
resistência mecânica e durabilidade natural da madeira (Silva \& Oliveira, 2003).

\subsubsection{Densidade da madeira}

A densidade básica média encontrada no lote da espécie Cedrela odorata foi de $0,41 \mathrm{~g} / \mathrm{cm}^{3}$, e para a espécie de Cedrelinga cateniformis foi de $0,47 \mathrm{~g} / \mathrm{cm}^{3}$.

Lobão et al. (2010), estudando 15 espécies florestais, dentre elas a C. odorata, encontraram um valor de densidade básica média de $0,34 \mathrm{~g} / \mathrm{m}^{3}$. Este valor inferior ao encontrado pode estar associado a diversos fatores, como idade da árvore, local de plantio, tratos silviculturas, disponibilidade de nutrientes no solo, entre outros.

Dias \& Lahr (2004) encontraram valores médios de densidade básica de 0,51 e 0,56 para C. odorata e C cateniformis, respectivamente. Já Gonçalez \& Gonçalves (2001) encontraram para C. cateniformis um valor de densidade básica média de $0,47 \mathrm{~g} / \mathrm{cm}^{3}$, semelhante ao encontrado neste estudo.

A densidade básica é considerada um dos principais parâmetros de qualidade da madeira quando se visa sua utilização como matéria-prima industrial ou energética, e seu estudo vem sendo largamente realizado, por ser de simples determinação e se correlacionar com outras propriedades da madeira. Por exemplo, observa-se que a espécie que apresentou maior densidade básica foi também a que apresentou maior teor de umidade.

Uma vez verificada a classificação da madeira conforme sua densidade básica, os dois cedros se enquadram na classe de madeira leve, conforme classificação feita por Melo et al. (1990).

Bortoletto (2003) em seu estudo afirma que as espécies adequadas para laminação apresentam densidade básica entre $0,38 \mathrm{~g} / \mathrm{cm}^{3}$ e $0,70 \mathrm{~g} / \mathrm{cm}^{3}$, com preferência para as madeiras com densidade próxima de $0,50 \mathrm{~g} / \mathrm{cm}^{3}$. Dessa forma, as espécies em estudo podem ser utilizadas para este fim.

\subsubsection{Variação dimensional}

O resultado de contração, inchamento e fator de anisotropia das madeiras podem ser visualizados nas Tabelas 2 e 3. Observa-se que os baixos coeficientes de variação e desvio-padrão indicam que as espécies apresentam menor variabilidade e maior homogeneidade dimensional, com exceção para o sentido longitudinal para ambas as espécies.
Segundo Caixeta et al. (2003), a contração e inchamento na direção longitudinal é baixa e raramente ultrapassa $1 \%$ para as madeiras, No geral, os valores médios nessa direção variam de 0,1 a $0,2 \%$ para a maioria das espécies. Os valores de contração para a C. cateniformis estão de acordo com os encontrados por Gonçalez \& Gonçalves (2001). Segundo o autor, esse valor é considerado médio, indicando que a madeira deve ser serrada depois que sua umidade entra em equilíbrio com a do ambiente. Porém, quando observados o Fator de anisotropia e Fator de inchamento, verifica-se que o valor de contração é baixo e a madeira pode ser considerada de excelente qualidade.

A Cedrelinga cateniformis se mostrou mais estável dimensionalmente do que a Cedrela odorata. Esse conhecimento pode ser utilizado como subsídio para a indicação das madeiras para determinados usos, onde tal propriedade seja requerida.

Os valores de contração volumétrica, assim como o inchamento volumétrico, foram considerados médios para Cedrelinga cateniformis e forte para Cedrela odorata, de acordo com o critério de classificação de madeiras descrito por Sallenave (1955) apud Gonçalez (1993), onde são consideradas madeiras de baixa contração volumétrica aquelas que possuem valores entre $4 \mathrm{e} 9 \%$, média contração aquelas cujos valores encontram-se entre 9 e 14\%, e madeiras de alta contração as que apresentam valores entre 14 e $18 \%$.

O coeficiente de anisotropia dimensional de contração e inchamento é definido pela razão entre a deformação ocorrida na direção tangencial e a deformação na direção radial (T/R). Segundo Melo \& Siqueira (1992), a diferença entre as contrações nas direções radial e tangencial explica a maior parte dos defeitos que ocorrem durante a secagem da madeira, tais como rachaduras e empenamentos.

Quanto mais altas as diferenças entre contração tangencial e radial, maior risco de inviabilizar o emprego de espécies de madeiras para fins nobres, como móveis finos, esquadrias, barcos, aparelhos musicais, aparelhos esportivos, etc. Sendo assim, o conhecimento desse coeficiente se torna útil no estudo das madeiras, por poder indicar a melhor utilização, em relação à variação dimensional (Oliveira e al., 2010).

Os fatores de anisotropia de contração e de inchamento para $C$. odorata e para $C$. cateniformis encontram-se descritos na Tabela 3. Embora a Cedrela 
Tabela 2. Valores médios, desvio-padrão e coeficientes de variação, das contrações e inchamentos das madeiras de C. cateniformis e C. odorata.

Table 2. Mean values, standard deviations and coefficients of variation of shrinkages and swells of the C. cateniformis and C. odorata woods.

\begin{tabular}{|c|c|c|c|c|c|c|c|c|}
\hline \multirow{3}{*}{ Espécies } & \multicolumn{8}{|c|}{ Variação Dimensional } \\
\hline & \multicolumn{4}{|c|}{ Contração (\%) } & \multicolumn{4}{|c|}{ Inchamento (\%) } \\
\hline & Tang & Radial & Long & Vol & Tang & Radial & Long & Vol \\
\hline Cedrela odorata & 9,05 & 7,37 & 0,22 & 15,94 & 9,96 & 7,96 & 0,22 & 18,97 \\
\hline DP & 0,28 & 0,56 & 0,04 & 0,58 & 0,34 & 0,66 & 0,04 & 0,82 \\
\hline CV (\%) & 3,13 & 7,62 & 18,72 & 3,62 & 3,44 & 8,23 & 18,75 & 4,30 \\
\hline Cedrelinga cateniformis & 6,18 & 4,73 & 0,19 & 10,78 & 6,58 & 4,97 & 0,19 & 12,09 \\
\hline DP & 0,32 & 0,13 & 0,04 & 0,33 & 0,36 & 0,15 & 0,04 & 0,42 \\
\hline CV (\%) & 5,13 & 2,82 & 19,55 & 3,10 & 5,47 & 2,96 & 19,58 & 3,47 \\
\hline
\end{tabular}

Tabela 3. Valores médios, desvio-padrão e coeficientes de variação, do fator de anisotropia de contração e de inchamento das madeiras de C. odorata e C. cateniformis. Table 3. Mean values, standard deviations and coefficients of variation of the anisotropy of shrinkage and swelling of the factor of C. odorata and C. cateniformis wood.

\begin{tabular}{lcc} 
Espécies & $\begin{array}{c}\text { Fator de } \\
\text { Anisotropia de } \\
\text { contração }\end{array}$ & $\begin{array}{c}\text { Fator de } \\
\text { anisotropia de } \\
\text { Inchamento }\end{array}$ \\
\hline Cedrela odorata & 1,23 & 1,26 \\
DP & 0,10 & 0,11 \\
\hline CV (\%) & 8,11 & 8,76 \\
Cedrelinga & 1,31 & 1,33 \\
cateniformis & 0,08 & 0,08 \\
DP & 5,75 & 6,10 \\
\hline CV (\%) & & \\
\hline
\end{tabular}

tenha se mostrado mais instável dimensionalmente do que a Cedrelinga, ela revelou um menor Fator de Anisotropia de contração e de Inchamento, significando que apresenta menos defeitos na hora do processamento.

Segundo Oliveira et al. (2010), este índice é importante, pois informa que, quanto maior for o seu distanciamento da unidade, mais propensa será a madeira a fendilhar e empenar durante as alterações dimensionais provocadas pela variação higroscópica.

Pelas amostras e características anatômicas e físicas analisadas neste trabalho, considera-se possível uma correta identificação de amostras da madeira. E, uma vez comparadas, verifica-se a importância desses valores, principalmente por se tratar de madeiras muito utilizadas na fabricação de móveis no município de Cruzeiro do Sul.
As informações das propriedades físicas das madeiras podem auxiliar na adequada indicação da aplicação final, uma vez que a densidade básica se correlaciona com resistência mecânica, porosidade, facilidade de secagem e penetração de preservantes, poder calorífico, acabamentos externos, trabalhabilidade, laminação, rendimento de polpação e fator de compactação de painéis aglomerados (Batista et al., 2010).

\section{CONCLUSÕES}

Conclui-se com o presente estudo que:

- Para separação entre as espécies, a característica anatômica mais útil é o parênquima axial.

- As duas espécies são consideradas leves.

- A estabilidade dimensional das espécies é excelente e demonstra baixa susceptibilidade aos defeitos do processo de secagem.

- A contração volumétrica foi considerada média para Cedrelinga cateniformis e forte para Cedrela odorata.

- A madeira pode ser empregada no setor moveleiro para fabricação de móveis finos, esquadrias, barcos, aparelhos musicais, aparelhos de esporte, etc.

\section{STATUS DA SUBMISSÃO}

Recebido: 12 jun., 2014

Aceito: 17 mar., 2017

\section{AUTOR(ES) PARA CORRESPONDÊNCIA}

\section{Mara Lúcia Agostini Valle}

Centro de Formação em Ciências e Tecnologias Agroflorestais, Universidade Federal do Sul 
da Bahia - UFSB, Campus Jorge Amado, Rua Itabuna, s/n, Rod. Ilhéus, Vitória da Conquista, Km 39, BR 415, Ferradas, CEP 45613-204, Itabuna, BA, Brasil

e-mail:maraagostini@yahoo.com.br

\section{REFERENNCIAS}

Acre. Secretaria Executiva de Floresta e Extrativismo SEF. Diagnóstico do Setor Florestal Madeireiro do Estado do Acre. Rio Branco: SEF; 1999.15 p.

Alves C, Pereira KRM, Silva TTS, Frota VB, Souza IAT. Avaliação das propriedades físicas de oito espécies usadas em serrarias de Rio Branco, Acre. In: Anais do $4^{\circ}$ Congresso Florestal Paranaense [online]. 2012; Curitiba. Curitiba: APRE; 2012. p. 1-9. [citado em 2016 jun. 1]. Disponível em: http://malinovski.com.br/CongressoFlorestal/ Trabalhos/06-Tecnologia/TPF-Artigo-04.pdf

Araújo HJB, Silva IG. Lista de espécies florestais do Acre: ocorrência com base em inventários florestais. Rio Branco: Embrapa Acre, 2000. 77 p. Documentos n. 48).

Araújo HJB. Agrupamento das espécies madeireiras ocorrentes em pequenas áreas sob manejo florestal do Projeto de Colonização Pedro Peixoto (AC) por similaridade das propriedades físicas e mecânicas [dissertação]. Piracicaba: Escola Superior de Agricultura "Luiz de Queiroz", Universidade de São Paulo; 2002.

Associação Brasileira de Normas Técnicas - ABNT. NBR-7190: ações nas estruturas, propriedades da madeira e dimensionamento nos diferentes limites de utilização. Rio de Janeiro: ABNT; 1997.

Batista DC, Klitzke RJ, Santos CVT. Densidade básica e retratibilidade da madeira de clones de três espécies de Eucalyptus. Ciência Florestal 2010; 20(4): 665-674.

Bortoletto G Jr. Produção de compensados com 11 espécies do gênero Eucalyptus, avaliação das suas propriedades físico-mecânicas e indicações para utilização. Scientia Forestalis 2003; 63: 65-78.

Caixeta RP, Trugilho PF, Rosado SCS, Lima JT. Propriedades e classificação da madeira aplicadas à seleção de genótipos de Eucalyptus. Revista Árvore 2003; 27(1): 43-51. http:// dx.doi.org/10.1590/S0100-67622003000100006.

Calderon CMA. O segmento moveleiro na região do Alto Juruá - AC: Perfil e uso de tecnologias alternativas para a caracterização das principais espécies madeireiras [tese]. Brasília: Universidade de Brasília; 2012.

Carvalho A. Madeiras portuguesas: estrutura anatómica, propriedades, utilizações. Lisboa: Instituto Florestal, 1996. vol. 1.

Carvalho JOP, Santos JJ, Saldanha EB, Melo ASG, Nascimento SM, Hirai EH, et al. Identificação botânica de espécies arbóreas pouco conhecidas como contribuição ao planejamento do manejo de florestas naturais no estado do Pará. In: Anais da I Conferência do Subprograma de Ciência e Tecnologia; 2008; Belém. Brasília: CNPq; 2009. p. 579.

Catanhede CN, García JN, Díaz MP. Agrupamento de espécies madeireiras da Amazônia em função da densidade básica e propriedades mecânicas. Madera y Bosques 1997; 3(1): 33-52. http://dx.doi.org/10.21829/myb.1997.311378.

Coradin VTR, Muniz GIB. Normas e procedimentos em estudos de anatomia da madeira: I - Angiospermae, II - Gimnospermae. Brasília: IBAMA; 1992. 19 p. Série Técnica n. 15.

Dias FM, Lahr FAR. Estimativa de propriedades de resistência e rigidez da madeira através da densidade aparente. Scientia Forestalis 2004; 65: 102-113.

Ferreira GC, Gomes IJ, Hopikns GJM. Estudo anatômico das espécies de Leguminosae comercializadas no Estado do Pará como “angelim”. Acta Amazonica 2004; 34(3): 71-76.

Gatto DA, Santini EJ, Haselein CR, Durlo MA. Qualidade da madeira serrada na região da quarta colônia de imigração italiana do Rio Grande do Sul. Ciência Florestal 2004; 14(1): 223-233.

Gonçalez JC. Caracterisation technologique de quatre especes peu connues de la forêt Amazonienne: anatomie, chimie, couleur, propriétés physiques et mécaniques [Tese]. Nancy, France: Ecole Nationale dun Génie Rural, des Eaux et des Forêts; 1993.

Gonçalez JC, Gonçalves DM. Valorização de duas espécies de madeira Cedrelinga cateniformis e Enterolobium schomburgkii para a indústria madeireira. Brasil Florestal 2001; 70: 69-74.

Lobão MS, Chagas MP, Costa DSP, Ferreira ATB, Sette CR Jr,, Carvalho IL, et al. Agrupamento de espécies florestais pela similaridade das características físico-anatômicas e usos da madeira. Cerne 2010; 16(Supl.): 97-105. []

Logsdon NB, Finger Z, Penna JE. Caracterização físicomecânica da madeira de Cedro-marinheiro, Guarea trichilioides L. (Meliaceae). Scientia Forestalis 2008; 36(77): 43-51.

Mafra ES. Estudo de algumas propriedades de dez espécies de madeiras de folhosas tropicais [dissertação]. Viçosa: Universidade Federal de Viçosa; 1994.

Marcarti CR. Estudo da anatomia e das propriedades tecnológicas da madeira deangico vermelho (Piptadenia peregrina Benth.) [dissertação]. Viçosa: Universidade Federal de Viçosa; 1992.

Melo JE, Coradin VTR, Mendes JC. Classes de densidade para madeiras da Amazônia brasileira. In: Anais do Congresso Florestal Brasileiro; 1990; Campos do Jordão. São Paulo: Sociedade Brasileira de Silvicultura; 1990. p. 695-699.

Melo JE, Siqueira MJ. Correlação entre propriedades físicas e mecânicas de madeiras da Amazônia. In: Encontro Brasileiro em Madeiras e em Estruturas de Madeira; 1992; São Carlos. São Carlos: USP; EESC; SET; 1992. p. 67-76. 
Nogueira EM, Fearnside PM, Nelson BW. Normalization of wood density in biomass estimates of Amazon forests. Forest Ecology and Management 2008; 256(5): 990-996. http://dx.doi.org/10.1016/j.foreco.2008.06.001.

Oliveira JT, Tomazello M Fo, Fiedler NC. Avaliação da retratibilidade da madeira de sete espécies de Eucalyptus. Revista Árvore 2010; 34(5): 929-936. http://dx.doi. org/10.1590/S0100-67622010000500018.

Oliveira JTS, Hellmeister JC, Tomazello M Fo. Variação do teor de umidade e da densidade básica da madeira de sete espécies de eucalipto. Revista Árvore 2005; 29(1): 115-127. http://dx.doi.org/10.1590/S0100-67622005000100013.

Panshin AJ, Zeeuw C. Textbook of wood technology, structure, identification, properties, and uses of the commercial woods of the United States and Canada. 4th ed. New York: McGraw-Hill; 1980.

Razera A No. Espécies de madeiras tropicais brasileiras na produção de móveis com madeira sólida na Região de Curitiba e municípios vizinhos [dissertação]. Curitiba: Universidade Federal do Paraná; 2005.

Rezende MA, Saglietti JRC, Guerrini IA. Estudo das interrelações entre massa específica, retratibilidade e umidade da madeira do Pinus caribaea var. hondurensis aos 8 anos de idade. Revista IPEF 1995; 48/49: 133-141.

Silva JC, Oliveira JTS. Avaliação das propriedades higroscópicas da madeira de Eucalyptus saligna Sm., em diferentes condições de umidade relativa do ar. Revista Árvore 2003; 27(2): 233-239. 